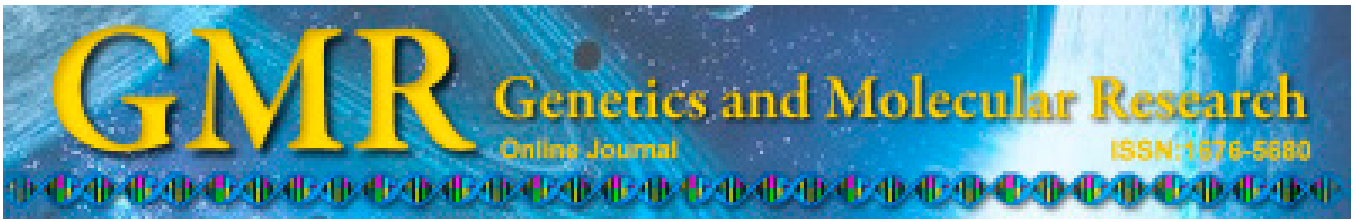

\title{
Apelin polymorphism predicts blood pressure response to losartan in older Chinese women with essential hypertension
}

\author{
J. Jia*, C. Men*, K.-T. Tang and Y.-Y. Zhan \\ Geriatric Medicine Department, \\ The First Affiliated Hospital with Nanjing Medical University, \\ Nanjing City, Jiangsu Province, China \\ *These authors contributed equally to this study. \\ Corresponding author: Y.Y. Zhan \\ E-mail: yiyangzhan@sina.com
}

Genet. Mol. Res. 14 (2): 6561-6568 (2015)

Received October 10, 2014

Accepted February 2, 2015

Published June 12, 2015

DOI http://dx.doi.org/10.4238/2015.June.12.10

\begin{abstract}
We determined whether the blood pressure response to losartan in an older Chinese population with essential hypertension was associated with apelin gene polymorphisms. We genotyped the $-1860 \mathrm{~T}>\mathrm{C}$ polymorphism of the apelin gene in a case-control study of 222 patients with hypertension and 250 controls. Following 24 weeks of treatment with losartan $(50 \mathrm{mg} /$ day), reductions in systolic blood pressure were significantly different among the additive (CT vs CC vs $\mathrm{TT}$ ), dominant (TT vs CC/CT), and recessive models (CC vs CT/TT; all $\mathrm{P}<0.05)$ in women but not in men. In the additive model, the TT group showed the greatest reductions in systolic BP $(23 \pm 10 \mathrm{mmHg})$ after treatment. The CT group showed greater reductions in systolic BP (21 $\pm 11 \mathrm{mmHg})$ compared to the $\mathrm{CC}$ group $(8 \pm 3 \mathrm{mmHg})(\mathrm{P}<0.05)$. The reductions in systolic $\mathrm{BP}$ of the TT and $\mathrm{CT} / \mathrm{CC}$ groups were $23 \pm 10$ and $19 \pm 10 \mathrm{mmHg}$, respectively. The reductions in systolic BP of the CC and TT/CT groups were $8 \pm 3$ and $21 \pm 10 \mathrm{mmHg}$, respectively. After adjustment for confounding factors, quantitative trait analysis with a
\end{abstract}


general linear model showed that the female patients with TT genotype showed greater reductions in systolic blood pressure after 24 weeks of treatment compared to the patients with the $\mathrm{C}$ allele $(\mathrm{P}<0.05)$. The apelin $-1860 \mathrm{~T}>\mathrm{C}$ genotype may play an important predictive role in the response to losartan in hypertensive women.

Key words: Apelin; Polymorphism; Losartan; Hpertension; Chinese

\section{INTRODUCTION}

Essential hypertension is a result of interactions influenced by both genetics and the environment. It is a highly prevalent disease and has been reported to occur in $39.1 \%$ of Chinese urban residents over 35 years of age (Meng et al., 2011). The renin-angiotensin system (RAS) plays a pivotal role in regulating blood pressure (BP), and many anti-hypertensive drugs target RAS. The individual response of patients to RAS inhibitors, however, is quite variable. Although the relationships between RAS gene polymorphisms and essential hypertension have been extensively studied, the roles of genes that modulate the RAS pathway have not been explored. Apelin and its receptor, apelin receptor (APJ), are two such examples.

Apelin was isolated in 1998 by Tatemoto et al. (1998) as a 36-amino acid peptide from bovine stomach extracts. The peptide was named apelin, since it was identified as the endogenous ligand to the orphan G-protein coupled receptor APJ. Apelin has high sequence homology to angiotensin II (Oudit et al., 2003). Plasma apelin levels are decreased in patients with hypertension, suggesting that apelin exerts a protective effect. The apelin $-1860 \mathrm{~T}>\mathrm{C}$ polymorphism was first found in the Han Chinese population by Li et al. (2009). The apelin -1860T $>\mathrm{C}$ is a newly identified Single nucleotide polymorphism (SNP) that has already been submitted to the GenBank with the NCBI number ss105110718. It is located in the promoter region of the apelin gene (Kidoya et al., 2008). The minor allele frequency of the -1860T>C SNP is 0.347 in the Chinese (Kidoya et al., 2008). The variation in SNP -1860T $>$ C may affect the transcription factor and transcriptional activity, which could alter the levels of apelin in the plasma. Based on this, we hypothesized that the $-1860 \mathrm{~T}>\mathrm{C}$ SNP may be associated with high BP and the response to antihypertensive agents.

The purpose of our study, therefore, was to examine whether apelin gene polymorphisms are associated with the risk of hypertension and response to losartan in the Han Chinese population.

\section{METHODS}

\section{Patient selection}

A total of 222 older patients with hypertension and 250 age-matched controls were randomly recruited from a community-based epidemiological survey in Jiangsu Province, China. Their ages ranged from 60 to 80 years. Both case and control populations were of Han ethnicity. The experimental protocol was approved by our institution review board, and all patients gave informed consent. All individuals with systolic BP (SBP) $\geq 140 \mathrm{mmHg}$ and/or diastolic $\mathrm{BP}(\mathrm{DBP}) \geq 90 \mathrm{mmHg}$ in consecutive measurements were selected for the case group. The exclusion criteria were as follows: treatment with antihypertensive agents; secondary hy- 
pertension; chronic diseases of the kidney or liver; diabetes mellitus; coronary artery disease; cerebrovascular disease; or cancer. Those free of hypertension (SBP $<140 \mathrm{mmHg}$ and $\mathrm{DBP}<$ $90 \mathrm{mmHg}$ ) were assigned to the control group. All hypertensive patients received therapy with losartan $(50 \mathrm{mg} /$ day $)$ for 24 weeks.

\section{BP and laboratory measurements}

BP was measured using a mercury sphygmomanometer. The average of three measurements taken after the patient rested for $15 \mathrm{~min}$ in a seated position was determined. Systolic and diastolic BP were measured before and after the 24-week treatment period. Participants were not permitted to smoke or drink alcohol or coffee in the hour before the BP measurement. Each participant was interviewed using a standardized questionnaire that included information on gender, age, height, weight, waist circumference, smoking, drinking, and hip circumference. Laboratory measurements included serum concentrations of blood urea nitrogen (BUN), blood creatinine $(\mathrm{Cr})$, low-density lipoprotein cholesterol (LDL-C), high-density lipoprotein cholesterol (HDL-C), total cholesterol (TC), triglycerides (TG), uric acid (UA), and fasting blood glucose (FBG).

\section{Genotyping}

Genomic DNA was extracted from leukocytes using the TIANGEN TIANamp Blood DNA Kit (TIANGEN, No. J8107, Beijing, China). The SNP in apelin was genotyped using the Multiplex SNaPshot technique. The primers and probes were designed using the primer 3.0 software (http://www.genome.wi.mit.edu/cgi-bin/primer/primer2.cgi). The forward primer of apelin T1860C was 5'-TGCTTTGTTCACAGCTGTGTC-3' and the reverse primer was 5'-ATATCCTGGGTCTCAAGGATG-3'. Multiplex polymerase chain reaction (PCR) was performed using $25 \mu \mathrm{L}$ at a final volume of $15 \mu \mathrm{L}$, which consisted of a reaction mixture containing $10 \times 0.15 \mu \mathrm{L}, 1.5 \mu \mathrm{L}$ buffer, $0.3 \mu \mathrm{L}$ dNTPs $(10 \mathrm{mM}), 0.9 \mu \mathrm{L} \mathrm{MgCl}_{2}(25 \mathrm{mM}), 0.1$ $\mu \mathrm{L}$ Taq DNA polymerase (TAKARA Biotechnology Co. Ltd., Dalian, China), $0.5 \mu \mathrm{L}$ of each primer $(10 \mathrm{pM})$, and $1 \mu \mathrm{L}$ DNA template $(20 \mathrm{mg} / \mathrm{L})$. The PCR procedure was performed as follows: initial denaturation at $95^{\circ} \mathrm{C}$ for $3 \mathrm{~min}$, denaturation at $94^{\circ} \mathrm{C}$ for $15 \mathrm{~s}$, annealing at $60^{\circ} \mathrm{C}$ for $15 \mathrm{~s}$, and recursive descent $0.5^{\circ} \mathrm{C}$, followed by extension at $72^{\circ} \mathrm{C}$ for $30 \mathrm{~s}$, for a total of 11 cycles. This was followed by 24 cycles of denaturation at $94^{\circ} \mathrm{C}$ for $15 \mathrm{~s}$, annealing at $54^{\circ} \mathrm{C}$ for $15 \mathrm{~s}$, extension at $72^{\circ} \mathrm{C}$ for $30 \mathrm{~s}$, and a final extension at $72^{\circ} \mathrm{C}$ for $3 \mathrm{~min}$.

For the snapshot reaction, we took the purified PCR products, each at a concentration of $0.2 \mu \mathrm{M} / \mathrm{L}$. We combined the snapshot primer and snapshot fluorescent mixtures. The snapshot response procedures included: initial denaturation at $96^{\circ} \mathrm{C}$ for $1 \mathrm{~min}$, denaturation at $96^{\circ} \mathrm{C}$ for $10 \mathrm{~s}$, annealing at $52^{\circ} \mathrm{C}$ for $5 \mathrm{~s}$, extension at $60^{\circ} \mathrm{C}$ for $30 \mathrm{~s}$, for a total of $30 \mathrm{cycles}$, and finally extension at $60^{\circ} \mathrm{C}$ for $30 \mathrm{~s}$. Amplified samples were stored at $4^{\circ} \mathrm{C}$. Snapshot PCR products, using Shrimp Alkaline Phosphatase (SAP) purification in $10 \mu \mathrm{L}$ snapshot PCR product with $1 \mathrm{U}$ SAP or $1 \mathrm{U}$ calf intestinal alkaline phosphatase (CIP), were mixed and insulated at $37^{\circ} \mathrm{C}$ for $45 \mathrm{~min}$ and at $80^{\circ} \mathrm{C}$ for $15 \mathrm{~min}$ to inactivate the enzyme. The samples were stored at $-20^{\circ} \mathrm{C}$. The results were analyzed with an ABI 3730XL Genetic Analyzer (Applied Biosystems, CA, USA) and the GeneMapper 4.0 software (Applied Biosystems, ABI, CA, USA). 


\section{Statistical analysis}

Data are reported as means \pm standard deviation (SD). Hardy-Weinberg equilibrium (HWE) was assessed by Fisher exact or chi-square $\left(\chi^{2}\right)$ test using the program HWE. Mean comparison between the two groups was analyzed by the $t$-test, and multiple group means were compared with one-way ANOVA and LSD for the post-test. The $\chi^{2}$ test or Fisher exact test was used for categorical variables. The general linear model (GLM) was applied to compare BP reductions among genotypes and was adjusted for covariates including age, body mass index (BMI), TG, TC, LDL-C, HDL-C, FBG, drinking, and smoking. All tests were twosided, and statistical significance level was set at $\mathrm{P}<0.05$. The data were statistically analyzed with the SPSS 13.0 (SPSS, USA).

\section{RESULTS}

The clinical characteristics of the participants (114 men, 108 women) and controls (128 men, 122 women) are shown in Table 1. There was no significant difference in terms of gender proportions between patients and controls.

Table 1. Baseline characteristics of the hypertensive and control groups.

\begin{tabular}{|c|c|c|c|c|c|c|}
\hline & \multicolumn{3}{|c|}{ Male } & \multicolumn{3}{|c|}{ Female } \\
\hline & Control & Hypertensive & $\mathrm{P}$ & Control & Hypertensive & $\mathrm{P}$ \\
\hline $\mathrm{N}$ & 128 & 114 & & & 122 & 108 \\
\hline Age (years) & $70 \pm 7$ & $69 \pm 7$ & 0.16 & $68 \pm 6$ & $67 \pm 5$ & 0.27 \\
\hline Body mass index & $23.4 \pm 2.26$ & $24.6 \pm 2.4$ & $<0.05$ & $23 \pm 3$ & $24.3 \pm 2.9$ & 0.014 \\
\hline Waist to hip ratio & $0.93 \pm 0.1$ & $0.92 \pm 0.06$ & 0.79 & $0.88 \pm 0.1$ & $0.89 \pm 0.07$ & 0.50 \\
\hline $\mathrm{SBP}(\mathrm{mmHg})$ & $122 \pm 10$ & $153 \pm 9$ & $<0.05$ & $116 \pm 14$ & $154 \pm 10$ & $<0.05$ \\
\hline DBP (mmHg) & $75 \pm 8$ & $86 \pm 10$ & $<0.05$ & $74 \pm 9$ & $86 \pm 10$ & $<0.05$ \\
\hline MBP (mmHg) & $91 \pm 7$ & $108 \pm 8$ & $<0.05$ & $88 \pm 8$ & $109 \pm 9$ & $<0.05$ \\
\hline $\mathrm{FBG}(\mathrm{mM})$ & $5.2 \pm 0.43$ & $4.96 \pm 0.4$ & $<0.05$ & $4.9 \pm 0.4$ & $5.0 \pm 0.5$ & 0.82 \\
\hline BUN (mM) & $6.09 \pm 1.01$ & $5.68 \pm 1.33$ & 0.008 & $6.09 \pm 4.4$ & $6.3 \pm 4.7$ & 0.68 \\
\hline Creatinine $(\mu \mathrm{M})$ & $89 \pm 30$ & $87.1 \pm 24.3$ & 0.50 & $88.1 \pm 29.3$ & $83.4 \pm 26.7$ & 0.20 \\
\hline Uric Acid $(\mu \mathrm{M})$ & $355.7 \pm 47$ & $330 \pm 56.9$ & $<0.05$ & $326.9 \pm 63$ & $324.8 \pm 67$ & 0.80 \\
\hline $\mathrm{TC}(\mathrm{mM})$ & $5.19 \pm 1.17$ & $5.2 \pm 1.13$ & 0.78 & $5.3 \pm 1.3$ & $5.2 \pm 1.03$ & 0.035 \\
\hline Triglycerides (mM) & $1.3 \pm 0.3$ & $1.38 \pm 0.42$ & 0.44 & $1.56 \pm 0.4$ & $1.48 \pm 0.42$ & 0.17 \\
\hline LDL (mM) & $2.61 \pm 0.67$ & $2.7 \pm 0.91$ & 0.31 & $2.81 \pm 0.92$ & $2.67 \pm 0.87$ & 0.23 \\
\hline HDL (mM) & $1.25 \pm 0.3$ & $1.3 \pm 0.3$ & 0.26 & $1.31 \pm 0.27$ & $1.4 \pm 0.3$ & 0.18 \\
\hline Heart Rate (bpm) & $74 \pm 8$ & $73 \pm 8$ & 0.16 & $72 \pm 8$ & $71 \pm 8$ & 0.48 \\
\hline Smoking $(\%)$ & $46 \%$ & $56.1 \%$ & 0.12 & $8.1 \%$ & $5.6 \%$ & 0.43 \\
\hline Drinking (\%) & $24.2 \%$ & $17.5 \%$ & 0.20 & $8.1 \%$ & $2.8 \%$ & 0.076 \\
\hline
\end{tabular}

Values are reported as means \pm SD and were analyzed using the $t$-test. The percentages of smokers and drinkers were analyzed using the Chi-square test.

The genotype/allele distributions of the apelin gene -1860T $>$ C polymorphism are depicted in Table 2. The apelin SNP conformed to the Hardy-Weinberg equilibrium in both control and case groups. Because the apelin gene is located on the X chromosome, we only presented the allele data for men. Apelin $-1860 \mathrm{~T}>\mathrm{C}$ genotype and allelic frequencies did not differ significantly between the hypertensive patients and controls.

After 24 weeks of treatment with losartan, significant reduction in systolic, diastolic, and mean arterial BP were observed (all, $\mathrm{P}<0.05$ ). The baseline values of systolic, diastolic, and mean arterial pressures were $153 \pm 10,88 \pm 8$, and $109 \pm 7 \mathrm{mmHg}$, respectively. After 
treatment with losartan, the systolic, diastolic, and mean arterial BP were decreased to $134 \pm$ $11,79 \pm 8$, and $98 \pm 8 \mathrm{mmHg}$, respectively (all, $\mathrm{P}<0.05$; Figure 1).

Table 2. Analysis of the association between the SNP and hypertension.

\begin{tabular}{cccc}
\hline & Case $(\mathrm{N}=222)$ & Control $(\mathrm{N}=250)$ & OR $(95 \% \mathrm{CI})$ \\
\hline Female & $\mathrm{N}=108$ & $\mathrm{~N}=122$ & \\
TT & $58(53.7 \%)$ & $67(54.9 \%)$ & $0.88(0.57-1.35)$ \\
CT & $43(39.8 \%)$ & $45(36.8 \%)$ & \\
CC & $7(6.5 \%)$ & $10(8.3 \%)$ & \\
Male & $\mathrm{N}=114$ & $\mathrm{~N}=128$ & $0.93(0.52-1.67)$ \\
C & $27(23.7 \%)$ & $32(25 \%)$ & \\
T & $87(76.3 \%)$ & $96(75 \%)$ & 0.56 \\
\hline
\end{tabular}

Chi-square $\left(\chi^{2}\right)$ test or Fisher's exact test was used for categorical variables.

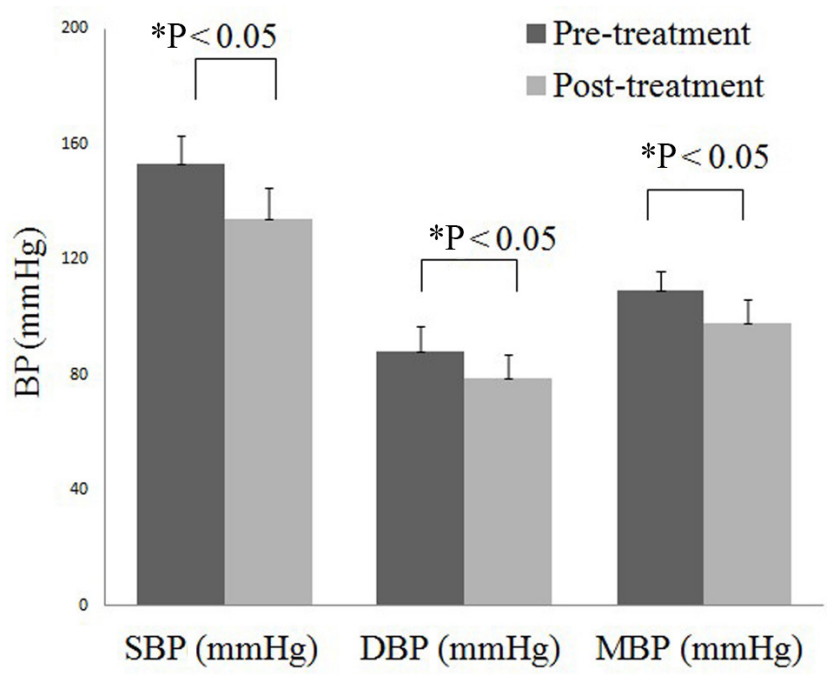

Figure 1. Blood pressure changes in hypertensive patients following treatment with losartan. The black bars represent the baseline values of systolic (SBP), diastolic (DBP), and mean arterial pressures (MAP), which were $153 \pm 10,88 \pm 8$, and $109 \pm 7 \mathrm{mmHg}$, respectively. The gray bars represent the SBP, DBP, and MAP that decreased to $134 \pm 11,79 \pm 8$, and $98 \pm 8 \mathrm{mmHg}$, respectively, after treatment with losartan. After treatment with losartan, significant reduction in SBP, DBP, and MAP was observed (all, $\mathrm{P}<0.05$ ).

In women, the reductions in systolic $\mathrm{BP}$ were significantly different in the additive (CT vs CC vs TT), dominant (TT vs CC/CT), and recessive models (CC vs CT/TT; all, $\mathrm{P}<$ $0.05)$. In the additive model, the TT group showed the greatest reductions in systolic BP (23 $\pm 10 \mathrm{mmHg})$ after treatment. The CT group showed greater reductions in systolic BP $(21 \pm 11$ $\mathrm{mmHg})$ compared to the $\mathrm{CC}$ group $(8 \pm 3 \mathrm{mmHg})(\mathrm{P}<0.05)$. The reductions in systolic $\mathrm{BP}$ of the TT and CT/CC groups were $23 \pm 10$ and $19 \pm 10 \mathrm{mmHg}$, respectively. The reductions in systolic $\mathrm{BP}$ of the $\mathrm{CC}$ and TT/CT groups were $8 \pm 3$ and $21 \pm 10 \mathrm{mmHg}$, respectively. After adjustment for confounding factors of age, BMI, TC, TG, HDL-C, LDL-C, FBG, drinking, and smoking, quantitative trait analysis with the GLM showed that the patients with TT genotype showed greater reductions in systolic BP after 24 weeks of treatment compared to the patients with the $\mathrm{C}$ allele $(\mathrm{CT}$ or $\mathrm{CC}$ genotype; $\mathrm{P}<0.05)$. The reductions in diastolic $\mathrm{BP}$ did not differ 
significantly in women, indicating similar responses to losartan (Table 3). Similarly, the reductions in systolic and diastolic BP did not differ significantly between the two male groups in the menmales, even after adjustment for covariates (all, $\mathrm{P}=$ not significant; Figure 2).

Table 3. Blood pressure reduction in relation to genotype in women.

\begin{tabular}{|c|c|c|c|c|c|c|c|}
\hline & \multicolumn{3}{|c|}{ Additive } & \multicolumn{2}{|c|}{ Dominant } & \multicolumn{2}{|c|}{ Recessive } \\
\hline & $\mathrm{CC}$ & $\mathrm{CT}$ & TT & TT & $\mathrm{CT} / \mathrm{CC}$ & $\mathrm{CC}$ & $\mathrm{TT} / \mathrm{CT}$ \\
\hline$\Delta \mathrm{SBP}(\mathrm{mmHg})$ & $8 \pm 3$ & $21 \pm 11$ & $23 \pm 10$ & $23 \pm 10$ & $19 \pm 10$ & $8 \pm 3$ & $21 \pm 10$ \\
\hline $\mathrm{F}$ & 6.278 & & & 4.548 & & 10.488 & \\
\hline $\mathrm{P}^{\mathrm{a}}$ & 0.003 & & & 0.035 & & 0.002 & \\
\hline$\triangle \mathrm{DBP}(\mathrm{mmHg})$ & $4 \pm 5$ & $8 \pm 7$ & $9 \pm 7$ & $9 \pm 7$ & $8 \pm 7$ & $4 \pm 5$ & $9 \pm 7$ \\
\hline $\mathrm{F}$ & 1.35 & & & 0.777 & & 2.481 & \\
\hline $\mathrm{P}^{\mathrm{a}}$ & 0.264 & & & 0.38 & & 0.119 & \\
\hline
\end{tabular}

aAdjusted for age, BMI, TC, TG, HDL-C, LDL-C, FBG, drinking, and smoking additive (CT vs CC vs TT), dominant (TT vs $\mathrm{CC} / \mathrm{CT}$ ), and recessive (CC vs $\mathrm{CT} / \mathrm{TT})$ models.

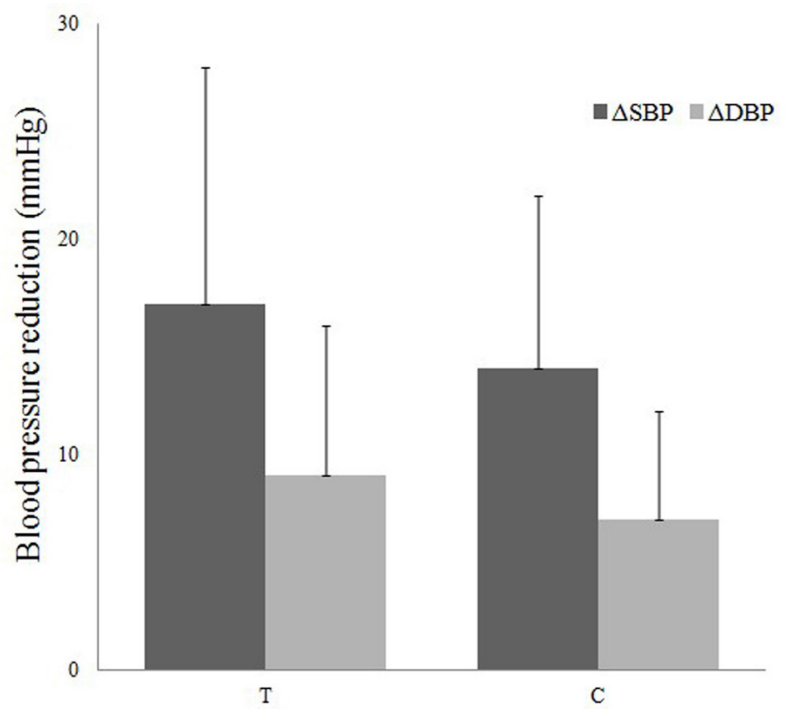

Figure 2. Blood pressure reduction in relation to genotype in men. The black bars represent the reductions in systolic blood pressure $(\triangle \mathrm{SBP})$. The gray bars represent the reductions in diastolic blood pressure $(\triangle \mathrm{DBP})$. In the $\mathrm{T}$ group, the $\triangle \mathrm{SBP}$ and $\triangle \mathrm{DBP}$ were $17 \pm 11$ and $9 \pm 7 \mathrm{mmHg}$, respectively. In the $\mathrm{C}$ group, the $\triangle \mathrm{SBP}$ and $\triangle \mathrm{DBP}$ were $14 \pm 8$ and $7 \pm 5 \mathrm{mmHg}$, respectively. The $\triangle \mathrm{SBP}$ and $\triangle \mathrm{DBP}$ did not differ significantly between the two male groups even after adjustment for age, BMI, TC, TG, HDL-C, LDL-C, FBG, drinking, and smoking (all P = not significant).

\section{DISCUSSION}

In this study, we present the novel finding of a genetic variant of the human apelin $(-1860 \mathrm{~T}>\mathrm{C})$ affecting the BP response to losartan. We also found that the apelin gene polymorphism is not associated with the risk of hypertension in older Chinese individuals. Our results demonstrate that older Chinese women with the apelin TT genotype had a greater response to the angiotensin II receptor antagonist losartan than patients with the $\mathrm{CC}$ and $\mathrm{CT}$ genotypes. 
This finding indicates the existence of a genetic component underlying the BP response to losartan and provides further clues for the mechanisms of the apelin-APJ system in the development of hypertension and response to treatment.

The current study is the first to investigate the relationship between a polymorphism of the apelin gene and the BP response to losartan in a Chinese population. It is already known that the BP response to losartan could be influenced by age, gender, race, and genetic factors; however, no study has been conducted previously to investigate the candidate genes for losartan-sensitivity of the BP response. In addition, older patients without previous hypertensive treatment were selected as participants to minimize the effects of confounding treatments. Second, the fact that the Han population is genetically homogenous increases the study power and internal validity. Finally, we provide new insights into the genetic variants of the apelin gene and sensitivity to losartan treatment.

The potential mechanisms of the apelin-APJ system in BP regulation are not fully understood. Previous studies have suggested that the Apelin-APJ system exerts a hypotensive effect through several different pathways. Apelin regulates blood vessel diameter through its cognate receptor, APJ, which is expressed on endothelial cells. Apelin enhances endothelial cell proliferation in the presence of vascular endothelial growth factor and promotes cell-tocell aggregation. The apelin/APJ system therefore regulates blood vessel diameter in part by stimulating angiogenesis (Kidoya et al., 2008). Apelin decreases the systemic secretion of arginine vasopressin, thereby inducing natural diuresis. In addition, the systemic administration of apelin decreases BP, improves cardiac contractility, and reduces cardiac loading (LlorensCortes and Kordon, 2008). The binding of apelin to APJ causes vasodilation via a nitric oxidedependent mechanism(Ishida et al., 2004).

In this study, the apelin gene was related to BP response to losartan in a sex-specific manner. Genetic variants of apelin had a significant effect on BP response in women but not in men. It is possible that the apelin gene has different effects on men and women because it is located on the X chromosome. Other genetic studies have reported the gender-specific effect of apelin in human diseases. For example, Liao et al. (2011) reported that in women, variants of the apelin gene were associated with obesity. In addition, the mechanisms underlying the $\mathrm{BP}$ response to losartan may be different between men and women. Sex-specific lifestyle differences in smoking and drinking incidence may explain some differences in the response to losartan. As apelin is located on the X chromosome, its genetic involvement in hypertension is likely to be even more prominent in women than in men. Moreover, sex hormones also exert direct effects and explain some of the differences in the incidence of hypertension between age-matched men, premenopausal women, and postmenopausal women. Stokes et al. (1989) and Fagard et al. (1995) reported that the APJ had a gender-specific function in peripheral immune activation of the hypothalamic-pituitary-adrenal axis, suggesting that the possible gender-specific influence of apelin/APJ on BP may involve differences in immune activation.

Polymorphisms in RAS genes are associated with the BP-lowering effect of losartan. Moore et al. (2007) found that a mutation in the renin gene influenced the hypotensive effect of losartan. Nakamura et al. (2004) demonstrated that the angiotensin-converting enzyme genotype may be a determinant of the response to losartan in hypertensive patients. Our study investigated a new $\mathrm{T}$ to $\mathrm{C}$ variation in the apelin promoter at position -1860 . The variation may affect transcription factor and transcriptional activity, which could lead to altered translation rates. These may explain the differences in the responses of patients with different genotypes to losartan.

Our study had some limitations. We did not measure plasma apelin levels, and there 
was potential for bias due to the relatively small sample size. Our findings, nonetheless, warrant future research into gender differences in the genetic mechanisms of the BP response to losartan.

In conclusion, we found that the apelin $-1860 \mathrm{~T}>\mathrm{C}$ genotype and allelic frequencies did not differ significantly between the participants with hypertension and controls. The apelin $-1860 \mathrm{~T}>\mathrm{C}$ polymorphism was associated with the BP response to losartan treatment in Chinese patients with hypertension. Women with the TT genotype were more sensitive to losartan treatment. The results of our study will provide guidance to clinicians involved in the treatment of hypertension that is resistant to losartan.

\section{Conflicts of interest}

The authors declare no conflict of interest.

\section{ACKNOWLEDGMENTS}

Research supported by the Priority Academic Program for the Development of Jiangsu Higher Education Institutions (Public Health and Preventive Medicine), the "Technology Project of Jiangsu Province" (\#BL2014085), and the "Six Elite Peaks" and "Innovative Team" project.

\section{REFERENCES}

Fagard R, Brguljan J, Staessen J, Thijs L, et al. (1995). Heritability of conventional and ambulatory blood pressures: a study in twins. Hypertension 26: 919-924.

Ishida J, Hashimoto T, Hashimoto Y, Nishiwaki S, et al. (2004). Regulatory roles for APJ, a seven-transmembrane receptor related to angiotensin-type 1 receptor in blood pressure in vivo. J. Biol. Chem. 279: 26274-26279.

Kidoya H, Ueno M, Yamada Y, Mochizuki N, et al. (2008). Spatial and temporal role of the apelin/APJ system in the caliber size regulation of blood vessels during angiogenesis. EMBO J. 27: 522-534.

Li WW, Niu WQ, Zhang Y, Wu S, et al. (2009). Family-based analysis of apelin and AGTRL1 gene polymorphisms with hypertension in Han Chinese. J. Hypertens. 27: 1194-1201.

Liao YC, Chou WW, Li YN, Chuang SC, et al. (2011). Apelin gene polymorphism influences apelin expression and obesity phenotypes in Chinese women. Am. J. Clin. Nutr. 94: 921-928.

Llorens-Cortes C and Kordon C (2008). Jacques Benoit lecture: the neuroendocrine view of the angiotensin and apelin systems. J. Neuroendocrinol. 20: 279-289.

Meng XJ, Dong GH, Wang D, Liu MM, et al. (2011). Prevalence, awareness, treatment, control, and risk factors associated with hypertension in urban adults from 33 communities of China: the CHPSNE study. J. Hypertens. 29: 1303-1310.

Moore N, Dicker P, O’Brien JK, Stojanovic M, et al. (2007). Renin gene polymorphisms and haplotypes, blood pressure, and responses to renin-angiotensin system inhibition. Hypertension 50: 340-347.

Nakamura Y, Tamaki S, Uchida Y, Ohmichi N, et al. (2004). Angiotensin converting enzyme genotype influences the response to the angiotensin II receptor antagonist losartan in patients with hypertension. Hypertens. Res. 27: 137-140.

Oudit GY, Crackower MA, Backx PH and Penninger JM (2003). The role of ACE2 in cardiovascular physiology. Trends Cardiovas. Med. 13: 93-101.

Stokes J 3rd, Kannel WB, Wolf PA, D’Agostino RB, et al. (1989). Blood pressure as a risk factor for cardiovascular disease. The Framingham Study-30 years of follow-up. Hypertension 13: 13-18.

Tatemoto K, Hosoya M, Habata Y, Fujii R, et al. (1998). Isolation and characterization of a novel endogenous peptide ligand for the human APJ receptor. Biochem. Biophys. Res. Commun. 251: 471-476. 